\title{
Management of postnatal depression
}

\author{
Charles Musters, ${ }^{1}$ Elizabeth McDonald, ${ }^{1}$ Ian Jones ${ }^{2}$
}

${ }^{1}$ East London NHS Foundation Trust, City and Hackney Centre for Mental Health, London E9 6SR

${ }^{2}$ Department of Psychological Medicine, University Hospital of Wales, Cardiff CF14 4XN

Correspondence to: C Musters charles.musters@eastlondon.nhs.uk

Cite this as: $B M J$ 2008;337:a736 doi:10.1136/bmj.a736
Depression is common in the postpartum period. It can be severe and may have serious consequences for the woman and her child. The term postnatal depression, however, is often applied indiscriminately to all occurrences of psychological distress after childbirth. It is important to identify and to treat postnatal depression-recently published National Institute for Health and Clinical Excellence (NICE) guidance supports a range of effective psychological and pharmacological interventions. ${ }^{1}$ Here, we discuss current thinking on the clinical features, aetiology, and management of postnatal depression.

\section{What is postnatal depression?}

Postnatal depression may form part of a bipolar or, more usually, a unipolar illness. Estimates vary but the overall prevalence of clinically relevant postnatal depression is $13 \%{ }^{2}$ Although classification systems consider onset within four weeks (Diagnostic and Statistical Manual of Mental Disorders) or six weeks (ICD-10; international classification of diseases, 10th revision) to be "postpartum," onsets within six months or even longer are usually included in clinical practice. Because several of the diagnostic features (box 1) such as tiredness, disturbed sleep, and reduced libidoare common perinatally, other symptoms-particularly feelings of worthlessness and hopelessness and early morning wakening before the baby-need to be sought. Suicidal thoughts and thoughts of harming the baby should specifically be asked about.

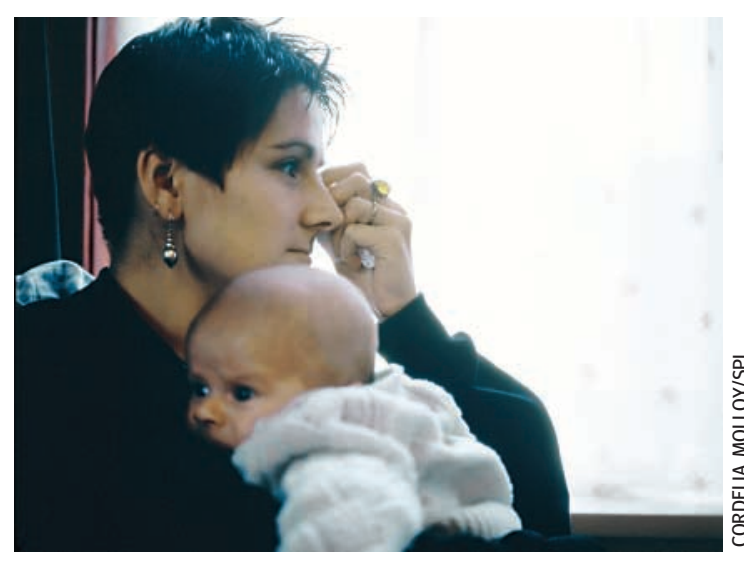

Postpartum blues or postnatal depression?

\section{What are the differential diagnoses?}

The main differential diagnoses are postpartum blues and postpartum psychosis. The blues affects at least $30 \%$ of women ${ }^{4}$; it causes transient emotional lability during the first week after birth and has a wide variety of symptoms typical of low and high mood. ${ }^{5}$ Care of the baby is not impaired, hopelessness and worthlessness are not prominent, and women do not feel suicidal. It is self limiting, but assessment should ensure that the woman is not and does not become more depressed.

The most severe form of postpartum mood disorder is puerperal (postpartum) psychosis. This condition is not easy to define, but the core feature is the acute onset of a manic or depressive psychosis soon after birth. ${ }^{6}$ This illness is a psychiatric emergency-patients are usually admitted and treated, preferably in a mother and baby unit. ${ }^{1}$ It occurs after around one in 1000 deliveries, but the risk is higher-in excess of one in two deliveries - in women with a history of bipolar disorder or puerperal psychosis. ${ }^{7}$ The tendency for all postpartum episodes to be labelled as postnatal depression can therefore have serious implications. The 2000-2 Confidential Enquiry into Maternal Deaths highlighted several suicides where previous postpartum psychosis was recorded at booking as postnatal depression, with suboptimal care being provided as a result. $^{8}$

\section{Is childbirth a specific trigger for episodes of depression?}

The psychosocial upheaval of childbirth and new motherhood is a potential risk factor for developing depression- $\mathrm{a}$ "non-specific trigger"-just one in a range of potential precipitants. However, a recent study in sisters with recurrent unipolar depression showed familial clustering of vulnerability, particularly for episodes with an onset within two months of birth. ${ }^{9}$ This suggests that childbirth may be a more specific "puerperal trigger" in a subgroup of women (box 2).

The nature of the puerperal trigger is still unknown, but several psychological, social, and biological factors probably play a part. Genetic factors have been implicated, ${ }^{9}$ and although most new mothers experience sleep deprivation, those who are most affected may be at higher risk. ${ }^{12}$ Many studies have looked at the role of hormones, but have found no major differences 


\section{Box 1 Summary of ICD-10 diagnostic criteria for depression ${ }^{3}$}

At least four, six, or eight symptoms are required for at least two weeks to make a diagnosis of mild, moderate, or severe depression, respectively. For mild and moderate depression, at least two group A symptoms must be present and for severe depression all three group $A$ symptoms are required.

\section{Group A symptoms}

Depressed mood to a degree that is abnormal for the person, present for most of the day, largely uninfluenced by circumstances

Loss of interest or pleasure in activities that are normally pleasurable

Decreased energy or increased fatiguability

\section{Group B symptoms}

Loss of confidence or self esteem

Unreasonable feelings of self reproach or excessive and inappropriate guilt

Recurrent thoughts of death or suicide, or any suicidal behaviour

Complaints or evidence of reduced ability to think or concentrate, such as indecisiveness

Change in psychomotor activity, with agitation or retardation

Sleep disturbance of any type

Change in appetite (decrease or increase) with corresponding weight change

in the hormonal physiology of women who develop postnatal depression. However a small double blind experimental study simulated the hormonal changes of pregnancy and the puerperium in parous women with and without a history of major depression and precipitated depressive symptoms only in women with a history of postnatal depression. ${ }^{13}$ This suggests that women who become depressed immediately postpartum may have an abnormal sensitivity to the normal physiological changes of childbirth.

\section{Who is at risk of postnatal depression?}

Key meta-analyses published in the past decade conclude that the principal risk factors for postnatal

\section{Box 2 Is postnatal depression distinct from non-postpartum depression?}

There has long been debate about whether postnatal depression is a distinct clinical entity. This debate has focused on whether the condition is clinically distinct from non-postpartum depression and whether depression is more prevalent after childbirth than at other times. Little evidence exists to suggest that the clinical features of postnatal depression are very different from depressive illness at other times of life. ${ }^{10}$ Several controlled studies over the past 15 years have not found an increased incidence of depression after childbirth. However, recent work suggests that women having children have fewer risk factors for depression; if this is taken into account, the postpartum period may indeed be a time of higher risk. ${ }^{11}$ The evidence does not therefore support postnatal depression as a separate clinical entity, but it does support the specific triggering of mood disorders by childbirth in at least a proportion of women.

\section{Box 3 The Whooley questions ${ }^{20}$}

- During the past month have you often been bothered by feeling down, depressed, or hopeless?

- During the past month have you often been bothered by having little interest or pleasure in doing things?

If the answer to either of these questions is positive, a third question is recommended by NICE

- Is this something you feel you need or want help with?

depression are depression or anxiety during pregnancy; a history of depression; a history of other psychiatric conditions; recent stressful life events; and poor social support, particularly the lack of a supportive partner. ${ }^{1415}$ The first of these risk factors highlights the importance of assessing and treating antenatal depression, and the possibility that a focus on postnatal illness may draw attention away from illness in pregnancy.

Although guidelines do not recommend routine screening to identify women at risk of postnatal depression, women at high risk of more severe episodes of postpartum mood disorder, including puerperal psychosis, should be identified. ${ }^{1}$ At booking or during pregnancy specific information should be sought, such as whether the woman has previously been treated by a psychiatrist; whether she has past or present severe mental illness including schizophrenia, bipolar disorder, psychosis in the postnatal period, and severe depression; and whether there is a family history of perinatal mental illness.

\section{Can postnatal depression be prevented?}

Studies of prophylactic drugs or psychological interventions do not support routine non-targeted interventions to reduce postpartum depression; however, intensive professional postpartum support individually targeted at at-risk mothers may be beneficial. ${ }^{16}$ Two small randomised clinical trials have examined antidepressant prophylaxis in pregnant women with a history of postnatal depression. A tricyclic antidepressant was not effective, ${ }^{17}$ but a selective serotonin reuptake inhibitor, sertraline, started after delivery reduced the risk of recurrence. ${ }^{18}$ Although the evidence base is scarce, it may be appropriate to offer antidepressant prophylaxis to some women with a strong history of depression.

Women on long term antidepressants who stopped treatment during pregnancy had a significantly increased risk of depressive relapse - $68 \%$ compared with $26 \%$ in women who continued their drugs. ${ }^{19}$ However, the women in this naturalistic study had longstanding and severe unipolar depression, so these figures may not hold for women with less severe forms of illness. Clearly, the risks and benefits of continuing drugs during pregnancy, even when a previous depressive episode has been treated successfully, need careful discussion with affected women.

For women with subthreshold depression, NICE guidelines recommend providing social support and brief structured psychotherapy (cognitive behavioural 


\section{Box 4 Assessing risk in postnatal depression}

A detailed mental state examination is a specialist assessment, but any clinician can sensitively ask about current symptoms of low mood; about ideas of worthlessness and hopelessness about the future; and about biological symptoms such as severe insomnia, poor appetite, and weight loss

Short term risks are of self neglect and neglect of the baby, further deterioration in the depressive illness, and suicide and infanticide

A preliminary assessment of the risks of suicide and infanticide can be made by asking about three key areas:

- The current mental state including thoughts of suicide and of harming the baby

- Any history of attempted suicide or harm to the baby

- Situational and environmental factors that might increase or decrease risk

When discussing symptoms of low mood, sensitively asking whether the woman feels that life is not worth living and whether she has ever thought of harming her baby enables the clinician to assess the most serious aspects of risk. Our experience is that mothers do not take offence if the context is right

Any psychotic symptoms substantially increase risk, particularly delusions or hallucinations that relate to the baby

The woman should be referred for a same day emergency appointment so that a detailed risk assessment can be carried out if self harm or harm to the baby are a possibility

therapy or interpersonal therapy), although these may not be available in all contexts

\section{How should I assess a woman with suspected postnatal depression?}

Depression should routinely and opportunistically be considered in all women in the perinatal period. The NICE guidelines recommend the Whooley questions as a screening tool (box 3), ${ }^{1}$ although they have not been investigated in the postnatal population. The Edinburgh postnatal depression scale is used more often, however. It has been psychometrically tested extensively in postpartum women and is used throughout the world. All of these are tools for screening, not diagnosis, and should be used only to identify women who need further clinical assessment.

Suspected mild and moderate depression can be assessed and managed in primary care. Women with severe depression or those who do not respond to treatment may need to be assessed by a psychiatrist, and referral should be urgent if there is a risk of self harm or harm to the child. Consider involving children and young persons' social services in such cases (box 4).

\section{How should I treat postnatal depression?}

NICE guidelines recommend a stepped care approach to the treatment of postnatal depression-self help strategies, non-directive counselling, brief cognitive behavioural therapy, and interpersonal psychotherapy are all recommended for mild to moderate depression. Antidepressants are recommended only if the patient declines psychological therapy, if it does not work, or if the woman has a history of severe depression. Even for severe depression, psychological treatment (cognitive behavioural therapy or interpersonal psychotherapy) is recommended as first line-antidepressants are prescribed only if requested by the patient, or if the response to psychological treatment is only partial.

The local availability of psychological therapies is an important factor when determining which treatment to offer, however, and waiting times can be lengthy. It is vital to treat women with severe illness promptly, even if drugs have to be used.

\section{Evidence for non-pharmacological treatments}

A recent Cochrane meta-analysis of 10 trials of psychological and psychosocial interventions concluded that peer support and non-directive counselling, cognitive behavioural therapy, psychodynamic psychotherapy, and interpersonal therapy are all effective in postnatal depression. ${ }^{21}$

\section{Evidence for pharmacological treatments}

Partly because of the ethical and practical problems of conducting drug trials in the perinatal period evidence on pharmacological management is limited. One trial randomised 87 women with postnatal depression to one of four groups - fluoxetine or placebo with either one or six sessions of cognitive behavioural counselling. ${ }^{22}$ All groups significantly improved, and improvement was significantly greater in the fluoxetine group than in the placebo group; however in this trial the refusal rate was high because many women did not want to take drugs. There is no evidence to suggest that treatments of benefit in non-postpartum depression are not also effective in postnatal depression. ${ }^{23}$

\section{Antidepressants and breast feeding}

In breastfeeding women the risk-benefit balance of antidepressant drugs is altered, with a consequent shift in emphasis towards psychological therapies. Treatment will depend on the patient's preference, previous

\section{Tips for non-specialists}

Women with postnatal depression may have contact only with non-specialists, so all health professionals who see pregnant and postnatal women need to be able to screen for the illness

The Whooley questions can be used as a brief and simple screening tool but have not been validated in this population. The Edinburgh postnatal depression scale and the hospital anxiety and depression scale can also be used without special training and take about five minutes to complete

Treatment can be effectively organised via primary care. Referral to specialist mental health services is advised for severe or high risk illness 
responses to treatment, local availability of psychological therapies, and the severity of the illness and the risks involved.

Certain antidepressants are considered safer than others in breastfeeding women, but in general the long term outcomes for exposed babies are unknown. Although tricyclic antidepressants have been prescribed for longer, most recent reproductive safety data, which include thousands of exposures, have been for selective serotonin reuptake inhibitors. Because of concerns about toxicity, tricyclics are prescribed less often than selective serotonin reuptake inhibitors for postnatal depression.

In a pooled analysis of 57 trials measuring concentrations of antidepressants in infant serum during breast feeding, ${ }^{24}$ fluoxetine and citalopram had the highest concentrations, and nortriptyline, sertraline, and paroxetine were undetectable. Because of lack of data, newer antidepressants, such as mirtazapine and venlafaxine, are not recommended for breastfeeding mothers.

Adverse effects may be greater in very young, premature, or systemically unwell babies. As with all drugs taken while breast feeding, the infant should be monitored regularly for sedation; irritability; and changes to sleep, feeding, and growth. New data are emerging regularly and up to date advice from specialist services may be useful in individual cases.

\section{What are the outcomes of postnatal depression?}

Unfortunately, differing definitions of postnatal depression across studies make it difficult to interpret the literature. Outcomes for women with very severe or psychotic episodes of depression are different from those for women with mild, subclinical depressive symptoms, and generalisations are not helpful.

Whatever the severity, however, most women who receive appropriate treatment completely recover but

\section{QUESTIONS FOR FUTURE RESEARCH AND ONGOING RESEARCH}

Little published evidence of the effectiveness of drug treatments in women with postnatal depression is available, and further research is needed in this area

Further work is needed to understand the triggering of mood disorders by childbirth, including the genetic and other determinants of vulnerability

Studies assessing whether effective treatment of antenatal depression can reduce the risk of postnatal depression are also needed

Results from two large randomised controlled trials in the UK will shortly be available. The PONDER (psychological interventions for postnatal depression) trial is assessing the effectiveness and cost effectiveness of two psychological interventions for postnatal depression delivered by health visitors. The RESPOND (randomised evaluation of antidepressants and support for women with postnatal depression) trial is comparing the effectiveness of antidepressants with counselling for postnatal depression delivered by health visitors

\section{ADDITIONAL EDUCATIONAL RESOURCES}

Additional resources for healthcare professionals National Institute for Health and Clinical Excellence (www. nice.org.uk)-Guidelines on antenatal and postnatal mental health

UK National Teratology Information Service (www.nyrdtc. nhs.uk/Services/teratology/teratology.html)—UK service providing up to date information on the risks and safety of drugs in pregnancy

Organisation of Teratology Information Services (www. otispregnancy.org)—US service providing up to date information on the risks and safety of drugs in pregnancy Milgrom J, Martin P, Negri L. Treating postnatal depression: a psychological approach for healthcare professionals. Chichester: Wiley, 2000

A two day training programme in perinatal psychiatry is held by the Southampton and New Forest Perinatal Mental Health Service in Winchester, Hampshire each year in June. Contact Sue Wallis (sue.wallis@hantspt-sw.nhs.uk)

Additional resources for patients

National Childbirth Trust (www.

nctpregnancyandbabycare.com)—UK charity for pregnant women and parents

SureStart (www.surestart.gov.uk) - This government programme to support young children and their parents runs a network of children's centres offering support, child health advice, and childcare

Royal College of Psychiatrists (http://www.rcpsych.ac.uk/ mentalhealthinformation/mentalhealthproblems/ postnatalmentalhealth/postnataldepression.aspx)_ Factsheet about postnatal depression

BBC online (www.bbc.co.uk/parenting/having_a_baby/ birth_pnd.shtml)—Factsheet about postnatal depression from the BBC's parenting website

Netmums (www.netmums.com)—Community support website for parents

Depression Alliance (www.depressionalliance.org) — UK charity for people with depression

Hanzac E. Eyes without sparkle: a journey through postnatal illness. Oxford: Radcliffe, 2005

Gilbert P. Overcoming depression. London: Constable and Robinson, 2000

are at risk of further postpartum and non-postpartum episodes. Some women are probably particularly vulnerable to depression related to childbirth. In a study of 55 primiparous women with postnatal depression and 40 controls followed over five years, ${ }^{25}$ women whose postnatal depression was their first episode of depression had a higher risk of subsequent postnatal depression $(41 \% v 18 \%)$ but a lower risk of non-postnatal depression (38\% v 62\%). On average, postnatal episodes lasted three to six months, but a substantial minority remained depressed at one year.

Clinical experience indicates that when postnatal depression has arisen in the context of clear psychosocial adversity, and has been mild or moderate in severity, the risk of subsequent depressive episodes 


\section{SUMMARY POINTS}

\section{Postnatal depression occurs after $13 \%$ of births}

A previous history of postnatal depression or of any mental illness, poor social support, and depression during the pregnancy all increase the risk of developing the illness

Postnatal depression needs to be identified and treated promptly and adequately because it can result in a range of lasting adverse outcomes for mother and child

A range of psychological therapies is effective in treating postnatal depression

Drugs are also effective and some antidepressants are thought to be safer in breastfeeding mothers than others

Drugs are recommended for women who decline psychological therapy, or for whom there would be an unacceptable delay in providing non-pharmacological measures

depends on the persistence of the adverse circumstances. If the adversity was discrete-such as the breakdown of a relationship - and has been resolved, then the risk of future postnatal depression may be low, but will depend on a wide range of factors particular to the woman. However, early onset depression (within the first six to eight weeks), severe depression, and depression with bipolar or psychotic symptoms may indicate a more specific puerperal triggering, and the risks of subsequent postpartum episodes may be higher. Advice about future risk should therefore be tailored to the individual woman.

Contributors: CM searched the literature and wrote the first draft. All authors helped with revision and agreed the final version. CM is guarantor. Competing interests: $\mathrm{CM}$ was paid the attendance fee for a conference by Janssen-Cilag. EM was paid an honorarium for chairing an academic meeting and the attendance fee and travel expenses for a conference by Janssen-Cilag. IJ has received honorariums or consultancy fees from Lilly, GlaxoSmithKline, Lundbeck, Sanofi-Aventis, Janssen-Cilag, and AstraZeneca and has received research funding from GlaxoSmithKline. Provenance and peer review: Commissioned; externally peer reviewed.

1 National Institute for Health and Clinical Excellence. Antenatal and postnatal mental health: clinical management and service guidance. Clinical guideline 45.

2007. www.nice.org.uk/guidance/index.jsp?

action $=$ bylD\&r=true \&o=11003.

2 O’Hara M, Swain A. Rates and risk of postpartum depression: a metaanalysis. Int Rev Psychiatry 1996;8:37-54.

3 WHO. International statistical classification of diseases and related health problems. 10th revision.

1993. www.who.int/classifications/icd/en/.

4 Harris B, Lovett L, Newcombe R, Read F, Walkler R, Riad-Fahmy D. Maternity blues and major endocrine changes: Cardiff puerperal mood and hormone study II. BMJ 1994;308:949-53.
5 Heron J, Craddock N, Jones I. Postnatal euphoria: are "the highs" an indicator of bipolarity? Bipolar Disord 2005;7:103-10.

6 Jones I, Craddock N. Bipolar disorder and childbirth: the importance of recognising risk. Br J Psychiatry 2005;186:453-4.

7 Munk-Olsen T, Laursen T, Pedersen C, Mors O, Mortensen P. New parents and mental disorders: a population-based register study. JAMA 2006;296:2582-9.

8 Lewis G, ed. The Confidential Enquiry into Maternal and Child Health (CEMACH). Why mothers die 2000-2002. The sixth report of the confidential enquiries into maternal death in the United Kingdom. London: RCOG Press, 2004.

9 Forty L, Jones L, Macgregor S, Caesar S, Cooper C, Hough A, et al. Familiarity of postpartum depression in unipolar disorder: results of a family study. Am J Psychiatry 2006;163:1549-53.

10 Cooper C, Jones L, Dunn E, Forty L, Haque S, Oyebode F, et al. Clinical presentation of postnatal and non-postnatal depressive episodes. Psychol Med 2007;37:1273-80.

11 Eberhard-Gran M, Eskild A, Tambs K, Samuelsen SO, Opjordsmoen S. Depression in postpartum and non-postpartum women: prevalence and risk factors. Acta Psychiatr Scand 2002;106:426-33.

12 Dennis C-L, Ross L. Relationships among infant sleep patterns, maternal fatigue, and development of depressive symptomatology. Birth 2005;32:187-93.

13 Bloch M, Schmidt P, Danaceau M, Murphy J, Nieman L, Rubinow D. Effects of gonadal steroids in women with a history of postpartum depression. Am J Psychiatry 2000;157:924-30.

14 Beck C. Predictors of postpartum depression: an update. Nurs Res 2001;50:275-85.

15 Robertson E, Grace S, Wallington T, Stewart D. Antenatal risk factors for postpartum depression: a synthesis of recent literature. Gen Hosp Psychiatry 2004;26:289-95.

16 Dennis C-L, Creedy D. Psychosocial and psychological interventions for preventing postpartum depression. Cochrane Database Syst Rev 2004;(4):CD001134.

17 Wisner K, Perel J, Peindl K, Hanusa B, Findling R, Rapport D. Prevention of recurrent postpartum depression: a randomized clinical trial. J Clin Psychiatry 2001;62:82-6.

18 Wisner K, Perel J, Peindl K, Hanusa B, Piontek C, Findling R. Prevention of postpartum depression: a pilot randomised clinical trial. $\mathrm{Am}$ J Psychiatry 2004;161:1290-2.

19 Cohen L, Altshuler L, Harlow B, Nonacs R, Newport D, Viguera A, et al. Relapse of major depression during pregnancy in women who maintain or discontinue antidepressant treatment. JAMA 2006;295:499-507.

20 Whooley M, Avins A, Miranda J, Browner W. Case-finding instruments for depression: two questions are as good as many. J Gen Intern Med 1997;12:439-45.

21 Dennis C-L, Hodnett E. Psychosocial and psychological interventions for treating postpartum depression. Cochrane Database Syst Rev 2007;(4):CD006116.

22 Appleby L, Warner R, Whitton A, Faragher B. A controlled study of fluoxetine and cognitive-behavioural counselling in the treatment of postnatal depression. BMJ 1997;314:932.

23 National Institute for Health and Clinical Excellence. Depression: management of depression in primary and secondary care. Clinical guideline 23 (amended). 2007. www.nice.org.uk/CG023NICEguideline.

24 Weissman A, Levy B, Hartz A, Bentler S, Donohue M, Ellingrod V, et al. Pooled analysis of antidepressant levels in lactating mothers, breast milk, and nursing infants. Am J Psychiatry 2004;161:1066-78.

25 Cooper P, Murray L. Course and recurrence of postnatal depression. Evidence for the specificity of the diagnostic concept. Br J Psychiatry 1995;166:191-5.

\section{What types of article does the $B M J$ consider?}

We are delighted to receive articles for publication-from doctors and others - on the clinical, scientific, social, political, and economic factors affecting health. We give priority to articles that will help doctors to make better decisions. Please see our advice to authors at http:// resources.bmj.com/bmj/authors, and if you would like to submit an article do so via our online editorial office at http://submit.bmj.com.

All original research articles are submitted, although we may invite submission (without promising acceptance) if we come across research being presented at conferences, if we see it in abstract form, or if the authors make an inquiry about the suitability of their work before submission.

We are also pleased to consider submitted articles for sections which carry a mix of commissioned and submitted articles - editorials, analysis, clinical review, practice, fillers, and Career Focus. Please follow the specific advice on each of these article types (see http:// resources.bmj.com/bmj/authors/types-of-article) before submitting your article. Some types of article - news, features, observations, head to head, views and reviewsare commissioned by the editors. 